\title{
Revue française de pédagogie
}

Recherches en éducation

164 | juillet-septembre 2008

Évaluation des politiques éducatives et comparaisons internationales

\section{La scolarisation d'élèves dyslexiques sévères en classe ordinaire de collège : lien entre adaptations pédagogiques, points de vue des enseignants et vécu de l'expérience scolaire des élèves}

Everely dyslexic students attending regular middle school classes: a bridge between educational adaptation policies, teachers' points of view and students' learning experience

die Einschulung schwer legasthenischer Schüler in eine gewöhnliche Klasse am Collège: Verbindung zwischen pädagogischer Anpassung, Standpunkt der Lehrer und erlebter Schulerfahrung der Kinder

Anne Gombert, Sylviane Feuilladieu, Pierre-Yves Gilles et Jean-Yves Roussey

\section{(2) OpenEdition Journals}

\section{Édition électronique}

URL : http://journals.openedition.org/rfp/2141

DOI : 10.4000/rfp.2141

ISSN : 2105-2913

Éditeur

ENS Éditions

Édition imprimée

Date de publication : 1 juillet 2008

Pagination : 123-138

ISBN : 978-2-7342-1136-5

ISSN : 0556-7807

Référence électronique

Anne Gombert, Sylviane Feuilladieu, Pierre-Yves Gilles et Jean-Yves Roussey, « La scolarisation d'élèves dyslexiques sévères en classe ordinaire de collège : lien entre adaptations pédagogiques, points de vue des enseignants et vécu de l'expérience scolaire des élèves », Revue française de pédagogie [En ligne], 164 | juillet-septembre 2008, mis en ligne le 01 juillet 2012, consulté le 19 avril 2019. URL : http://journals.openedition.org/rfp/2141; DOI : 10.4000/rfp.2141 


\title{
La scolarisation d'élèves dyslexiques sévères en classe ordinaire de collège : lien entre adaptations pédagogiques, points de vue des enseignants et vécu de l'expérience scolaire des élèves
}

\author{
Anne Gombert, Sylviane Feuilladieu, \\ Pierre-Yves Gilles \& Jean-Yves Roussey
}

Depuis la loi sur "l'égalité des droits et des chances des personnes handicapées" (2005-102, 02/2005) la notion d'intégration scolaire évolue vers celle d'inclusion: la société peut être handicapante et c'est à elle qu'incombe le devoir de s'adapter. Dans le cadre scolaire, l'adaptation par les enseignants de leurs pratiques professionnelles devient une condition sine qua non pour compenser les difficultés scolaires liées au handicap. Cependant, poser un cadre législatif ne suffit pas. La prise en charge de ces élèves, parfois très en deçà de la norme de la classe, constitue pour l'enseignant un véritable défi, en regard des multiples remises en cause que cela implique (Belmont \& Vérillon, 2003 ; Gombert \& Roussey, 2007). Se posent alors diverses questions relatives à ces gestes : quelles sont la nature et les fonctions des gestes que les enseignants mettent en place pour aider les élèves? Est-ce que les types de gestes varient en fonction du niveau d'enseignement (primaire ou secondaire) ? Observe-t-on une corrélation entre la manière dont les enseignants définissent le handicap, l'intégration scolaire et la dyslexie, et certains types de gestes ? Certains d'entre eux sont-ils, plus que d'autres, associés à un ressenti positif de l'intégration chez l'élève ? La recherche présentée s'attachera à répondre à ces questions en établissant un lien entre trois éléments: (1) la nature des gestes d'aide que les enseignants du primaire et du secondaire ont mis en œuvre lorsqu'ils ont intégré dans leurs classes des élèves souffrant d'un trouble spécifique sévère du langage écrit, (2) l'évocation des enseignants sur le handicap, l'intégration et le trouble spécifique (dyslexie et dysorthographie sévère), (3) la manière dont les élèves intégrés vivent leur expérience scolaire (Dubet \& Martucelli, 1996).

Descripteurs (TEE) : handicap, intégration scolaire, adaptations pédagogiques, point de vue des enseignants, expérience scolaire, trouble spécifique du langage écrit.

\section{SCOLARISATION DES ÉLÈVES EN SITUATION DE HANDICAP ET PRATIQUES ENSEIGNANTES}

La Classification internationale du fonctionnement, du handicap et de la santé (OMS, 2002) définit le handicap comme le résultat d'une interaction entre un problème de santé (maladie, trouble, traumatisme) et des facteurs contextuels liés à la personne (sexe, âge, origine sociale, profession...) mais aussi à l'environnement (attitudes sociales, architecture, 
législation...). Dans ce modèle conceptuel, intégrant à la fois des dimensions médicales et sociales, le handicap manifeste un dysfonctionnement pouvant toucher plusieurs niveaux: celui du corps, il exprime alors une déficience (atteintes de fonctions organiques ou de structures anatomiques) ; celui relatif à l'action de la personne (exécution d'une tâche), il implique une limitation d'activité ; et enfin, celui de la contribution de la personne à la vie sociale, il désigne une restriction de participation. Le handicap est donc considéré à la fois dans ses dimensions individuelle (prises en charge thérapeutiques) et sociétale (acceptation, aménagements matériels, prise en charge scolaire, sociale, financière...). La prise en compte de l'aspect sociétal est historiquement très récente. C'est à partir de la loi de 1975 en faveur des personnes handicapées et de la Classification internationale des handicaps (OMS, 1980) que le handicap est défini comme un désavantage social, subi par l'individu en conséquence de ses déficiences. Cette reconnaissance de la responsabilité collective et institutionnelle dans la situation de handicap est largement confirmée par la loi du 11 février 2005 « Pour l'égalité des droits et des chances, la participation et la citoyenneté des personnes handicapées ". Cette loi pose le principe d'une accessibilité généralisée à l'ensemble des domaines de la vie publique, et réaffirme le droit à la scolarisation des enfants handicapés dans l'école ordinaire de leur quartier. II ne s'agit plus seulement d'intégrer certains enfants handicapés dans certaines classes, mais de tous les scolariser et ceci, de préférence en classe ordinaire. Ce principe marque une évolution majeure. En effet, en un siècle le modèle ségrégationniste (de 1909 à 1975) a cédé la place au modèle intégratif (mis en œuvre à partir de 1975), lui-même évoluant lentement vers le modèle inclusif (Plaisance \& Gardou, 2001). Dans cette dernière conception, non seulement la société a le devoir de scolariser tous les enfants handicapés, mais elle a également celui de s'adapter à leurs besoins éducatifs particuliers (1) afin d'optimiser les chances de réussite du processus intégratif. Parce que la société peut être handicapante, il lui incombe le devoir de compensation, devenu d'ailleurs un droit pour la personne handicapée avec la loi de 2005. Ainsi, le modèle inclusif place la compensation du handicap au centre des politiques sociales et scolaires. II s'agit de mettre en œuvre des actions visant à rééquilibrer les désavantages sociaux qu'éprouve une majorité de personnes handicapées, afin de leur permettre l'égalité d'accès aux différents lieux et pratiques sociales. Autrement dit, corriger le constat des inégalités d'accès liées à la présence du handicap, par des mesures différenciées. Dans le cadre de l'école, les gestes pédagogiques adaptés constituent un moyen de compensation.

Pour autant, poser un cadre législatif ne suffit pas. Aujourd'hui encore à l'école, la prise en charge des élèves en situation de handicap, qui sont parfois très en deçà de la norme de la classe, constitue une réelle difficulté pour les enseignants (Belmont \& Verillon, 2003 ; Gombert \& Roussey, 2007). Trois raisons peuvent expliquer la persistance des obstacles à la scolarisation en classe ordinaire.

Premièrement sur le plan de la socialisation, inclure physiquement un enfant handicapé à l'école n'implique pas obligatoirement son intégration scolaire et sociale. La présence d'un enfant handicapé n'entraîne pas forcément une adaptation pédagogique de l'enseignant, sa réussite dans les apprentissages ou des relations avec ses pairs (Rousseau \& Belanger, 2004). Dans une étude qui portait sur des handicaps divers (déficience visuelle, auditive, motrice, trisomie, trouble de la conduite et du comportement), Zaffran (1997) observe une baisse quantitative et qualitative des interactions entre élèves en défaveur de l'élève handicapé. La confrontation à l'autre, lorsqu'il est très différent de la norme, génère des réactions de retrait voire de rejet, conséquences de la peur qu'il inspire et de la méconnaissance de ses capacités à être comme tout le monde (Goffman, 1975). Par exemple, dans le cas de l'autisme, c'est le code de communication qui prévaut à tout engagement dans une relation sociale qui n'est plus adéquat. Dans un autre registre, en situation ordinaire un enfant qui veut jouer avec un autre enfant, dans une cour de récréation par exemple, sait globalement: qu'il peut aller jouer avec lui sans se mettre en danger, sans mettre l'autre en danger, comment lui proposer ce jeu, comment l'autre va réagir... En présence d'un élève ayant des troubles du comportement ou infirme moteur cérébral par exemple, cette connaissance pratique n'est plus opérationnelle et entraîne soit une absence de relation, soit une gêne dans la relation qui peut se traduire par une attitude de surprotection ou d'agressivité. D'autres recherches montrent que des élèves porteurs de handicap (ici d'un trouble envahissant du développement) peuvent tirer bénéfice des échanges sociaux mixtes, à travers la confrontation à leurs pairs non handicapés et leur observation, pour peu qu'ils possèdent " certaines compétences " (Rivard \& Forget, 2006 ; Poirier et al., 2005). Ces recherches tendraient à montrer que certains enfants seraient plus " intégrables " que d'autres en fonction de leur handicap. Certains auteurs sont plus nuancés. Citons par exemple Robertson et al. (2003) ou encore 
Pianta et al. (1995), qui montrent que les "solutions pédagogiques » et l'attitude de l'enseignant peuvent faire tomber les peurs et favoriser les échanges. Ou bien Belmont \& Verillon (2003) dont le travail sur le partenariat entre les différents acteurs autour de l'élève témoigne de l'impact positif de la collaboration sur la réussite du processus intégratif. À l'instar de ces auteurs, nous pensons que la réussite du processus d'intégration scolaire relève de multiples facteurs, au-delà même du type de handicap.

Deuxièmement, sur le plan des apprentissages, le défi pour l'enseignant est de prendre en compte la diversité de tous les élèves (par exemple, des particularités liées à une déficience), tout en repérant les invariants pédagogiques susceptibles d'être exploités par l'ensemble du groupe. La loi de 2005 sur le handicap, ainsi que les textes d'application de cette loi dans l'Éducation nationale, stipulent clairement que l'adaptation pédagogique des enseignants est une condition sine qua non de la prise en charge des élèves en situation de handicap. Tous les aménagements sont possibles dès lors qu'ils visent à compenser les difficultés scolaires liées au handicap. Ils doivent être consignés dans le projet personnalisé de scolarisation de l'élève concerné (Circulaire 2006126 du 17 août 2006). Dans cette optique, les gestes d'adaptation pédagogique deviennent alors la clé de voûte de cette prise en charge, lorsque le handicap implique des difficultés dans les apprentissages scolaires. Ainsi, parmi l'ensemble des aides que I'on peut apporter aux élèves intégrés pour compenser leur handicap (auxiliaire de vie scolaire, tuteur, mise à disposition de matériel informatique, etc.), une des moins visibles est bien celle que l'enseignant fournit dans sa classe en mettant en œuvre une pédagogie différenciée (Peslouan \& Rivalland, 2003). À ce propos, Gombert \& Roussey (2007) ont montré que l'adaptation des pratiques enseignantes en vue de favoriser l'intégration d'élèves dyslexiques sévères ne relève pas de l'élaboration de solutions exceptionnelles mais plutôt de gestes élémentaires (simplification et reformulation des consignes de travail, adaptation des supports d'apprentissage et d'évaluation, adaptation des barèmes d'évaluation...). Pour autant, malgré cette simplicité apparente, leur mise en œuvre réfléchie représente pour l'enseignant une sorte de " mini-révolution" dans sa manière de concevoir la prise en charge des difficultés d'apprentissages dans sa classe. Cette mise en œuvre résulte d'un processus plus ou moins long, s'inscrivant dans le temps. La prise en compte des élèves aux besoins éducatifs particuliers (comme ceux porteurs de handicap) répond à deux principes : être attentif à la différence comme principe d'action, et ne pas opposer groupe classe et dispositif d'intégration (Thomazet, 2006). Dans la même optique, pour Anna (2003), la présence d'un élève lourdement handicapé en classe ordinaire exige de la part d'un enseignant: (a) d'accepter de ne pas savoir, de tâtonner, de se tromper ; (b) d'observer "autrement » (par exemple, de regarder les compétences plus que les performances); (c) d'être très patient et de savoir attendre ; (d) d'accepter les moindres progrès, les micro-réponses, d'accepter même que ce soient les seuls objectifs; (e) d'accepter que les apprentissages scolaires constituent une partie seulement de l'action, en complément de l'aspect de socialisation. La prise en charge scolaire d'un élève en situation de handicap n'interroge donc pas seulement le métier d'enseignant en termes de connaissances et de compétences (certes elles sont essentielles), elle interroge aussi l'identité professionnelle de l'enseignant (sa conception du métier, ses représentations de l'élève et des savoirs). C'est donc à la fois les aspects pratiques et théoriques de la profession qui sont questionnés.

Troisièmement le modèle inclusif du handicap, relayé par la loi de 2005, remet en cause une croyance majoritairement partagée, un « topoï », d'après Plantin (1993) : les élèves différents sont bien mieux dans des classes spécialisées. Cette croyance est l'héritière du mode de socialisation réservé aux personnes handicapées en France depuis le $\mathrm{XVII}^{\mathrm{e}}$ siècle. Historiquement, cela fait quatre siècles que les enfants handicapés sont placés et éduqués en instituts spécialisés (pour une revue sur l'historique de l'enseignement spécialisé en France, voir Gillig, 2006). De ce fait, et parce que l'impact des représentations sociales des professeurs sur leurs pratiques professorales est observé (Lautier, 2001, Felouzis, 1997), il apparaît essentiel de s'interroger sur la manière dont ils définissent le handicap et l'intégration dans le cadre de la scolarisation des élèves handicapés. L'évolution rapide et récente du cadre législatif n'implique pas forcément une évolution rapide des mentalités, même si l'image du handicap se modifie, à force d'opérations médiatiques et d'obligations citoyennes.

\section{UNE RECHERCHE EN PRIMAIRE ET COLLÈGE}

\section{Questions posées}

Dans ce contexte national, la question des gestes professionnels d'aide que les enseignants développent lorsqu'ils accueillent un élève porteur de 
handicap en classe ordinaire devient centrale. Les gestes d'adaptation pédagogique peuvent être mis en rapport avec la notion d'étayage telle que Bruner la définit $(1983,1984)$. Largement lié au concept de zone proximale de développement développé par Vygotski (Schneuwly \& Bronckart, 1985), l'étayage désigne "l'ensemble des interactions d'assistance de l'adulte permettant à l'enfant d'apprendre à organiser ses conduites afin de pouvoir résoudre seul un problème qu'il ne savait pas résoudre au départ " (Bruner, 1983). L'auteur en décrit six fonctions :

- l'enrôlement (c'est-à-dire engager l'intérêt et l'adhésion de l'enfant dans la tâche) ;

- la réduction des degrés de liberté (impliquant la simplification de la tâche de façon à la mettre à la portée de l'apprenant) ;

- le maintien de l'orientation (qui comprend par exemple le fait de proposer des tâches motivantes pour garder l'enfant mobilisé dans la tâche) ;

- la signalisation des caractéristiques déterminantes (c'est-à-dire l'ensemble des gestes qui signalent à l'enfant les éléments pertinents pour résoudre la tâche) ;

- le contrôle de la frustration (qui suppose la mise en œuvre d'actions permettant d'éviter que les difficultés rencontrées ne se transforment en échec et n'entraînent un sentiment de démotivation) ;

- la démonstration ou "présentation de modèles " (c'est-à-dire exécuter la tâche et faire avec l'élève à partir par exemple de ses propositions).

Se posent alors diverses questions relatives aux gestes étayants des enseignants: quelles sont la nature et les fonctions des gestes que les enseignants mettent en place pour aider les élèves ? Est-ce que les types de gestes varient en fonction du niveau d'enseignement (primaire ou secondaire) ? Observet-on une corrélation entre la manière dont les enseignants définissent le handicap, l'intégration scolaire et la dyslexie, et certains types de gestes ? Certains d'entre eux sont-ils, plus que d'autres, associés à un ressenti positif de l'intégration chez l'élève?

La recherche présentée s'attachera à répondre à ces questions en établissant un lien entre trois éléments :

- la nature des gestes d'aide que les enseignants du primaire et du secondaire ont mise en œuvre lorsqu'ils ont intégré dans leurs classes des élèves souffrant d'un trouble spécifique sévère du langage écrit ;
- l'évocation des enseignants sur le handicap, l'intégration et le trouble spécifique (dyslexie et dysorthographie sévère) ;

- la manière dont les élèves intégrés vivent leur expérience scolaire (Dubet \& Martucelli, 1996).

\section{Enquête de terrain}

Depuis le «plan d'action pour les enfants atteints d'un trouble spécifique du langage oral ou écrit » (2), la dyslexie/dysorthographie, diagnostiquée comme sévère, est considérée comme un handicap. Dans le cadre d'un dispositif pilote mis en place dans le département des Hautes-Alpes à la rentrée 2003, 24 élèves dyslexiques sévères (12 en primaire et 12 en collège) ont bénéficié pendant deux années scolaires d'une double prise en charge Santé - Éducation nationale. Certains de ces élèves étaient alexiques, d'autres étaient déscolarisés ou scolarisés dans des établissements spécialisés non adaptés à la prise en charge de leur trouble. Dans ce dispositif, ils étaient accueillis d'une part à temps partiel dans une maison d'enfants à caractère sanitaire, où ils bénéficiaient de soins thérapeutiques adaptés. D'autre part, ils étaient scolarisés à mi-temps dans des classes ordinaires (3). En primaire cette intégration était effectuée sur la base d'une demi-journée de présence (soit le matin, soit l'après midi) et, en collège, selon les disciplines. Ainsi, le curriculum scolaire de l'élève se trouvait modifié de fait, puisqu'il n'effectuait pas toutes les disciplines au bénéfice des soins dans le centre. Afin de privilégier l'aspect socialisant de la scolarisation en milieu ordinaire, l'intégration s'est faite sur la base d'un élève par classe, en fonction de son âge biologique et non de son niveau scolaire effectif. Par exemple, les trois élèves intégrés en classe de $5^{\mathrm{e}}$ avaient une compétence en lecture proche d'élèves de CE1 et, par voie de conséquence, un niveau scolaire largement en deçà de leur groupe classe.

Au $2^{\mathrm{e}}$ trimestre de la première année de la prise en charge, des entretiens semi-directifs ont été effectués auprès de trente enseignants du secondaire et huit professeurs des écoles (cf. annexe méthodologique $n^{\circ} 1$ ). Pour le secondaire, un large éventail de disciplines est représenté dans l'échantillon : 6 enseignants de français, 6 de mathématiques, 5 d'histoiregéographie, 3 de sciences de la vie et de la Terre, 3 de technologie, 3 d'éducation physique et sportive, 1 d'allemand, 1 de sciences physiques, 1 d'éducation musicale, 1 d'arts plastiques. Ces enseignants ont été interrogés sur leurs pratiques, les difficultés qu'ils ont rencontrées dans cette prise en charge, et leur 
définition du handicap, de la dyslexie, de l'intégration. À la fin de cette première année, des entretiens semi-directifs ont également été effectués auprès de 17 élèves ( 7 en primaire et 10 en secondaire, cf. annexe méthodologique $n^{\circ} 2$ ). Ils ont été interrogés sur les conséquences scolaires et sociales de leur prise en charge en classe (comment se sentent-ils en classe ? Pensent-ils mieux réussir dans les apprentissages ? Quelles différences perçoivent-ils avec leur scolarité antérieure?)

\section{Méthodologie}

Afin d'étudier la nature des gestes professionnels d'aide des enseignants, tous les énoncés concernant les pratiques pédagogiques d'aide à destination de l'élève intégré ont été relevés, qu'il s'agisse des manières de faire, des modifications pédagogiques, des dispositifs spécifiques, etc. Les 266 propositions ainsi répertoriées ont fait l'objet d'une analyse de contenu, à partir de laquelle a été élaborée une typologie des gestes d'aide (Gombert \& Roussey, 2007). Cette catégorisation a été constituée en respectant 5 principes de classement (Bardin, 1989). Les catégories doivent être :

- exhaustives (tous les énoncés du corpus à classer doivent être distribués dans les catégories);

- exclusives (un même élément ne doit pas être classable dans deux catégories ou sous-catégories à la fois) ;

- méthodiques (construites en fonction de règles strictes, explicitables et transmissibles à autrui) ;

- objectives (le tri doit être le plus objectif possible, ce qui nécessite de recourir à plusieurs codeurs) ;

- quantifiables (la catégorisation doit aboutir à des calculs aussi précis que possible). Les propositions ont été classées selon leur idée principale puis regroupées en "blocs" d'idées. Ces blocs d'idées représentent les catégories générales de la typologie. À l'intérieur de chaque catégorie, une hiérarchisation des informations a permis d'établir des "sous-catégories", qui préservent et regroupent des informations plus spécifiques. Le tableau 1 présente cette typologie : les catégories, le type de gestes professionnels classés dans ces catégories, ainsi que quelques exemples concrets de pratiques pédagogiques.

Pour approcher les représentations des enseignants, seules les réponses à trois questions ont fait l'objet de l'analyse: "Pour vous, c'est quoi intégrer? Pour vous, c'est quoi la dyslexie ? Pour vous, c'est quoi le handicap ? " (cf. annexe méthodologique $n^{\circ} 3$ ). L'unité retenue est l'ensemble des énoncés (bloc réponse) produits à l'issue de chacune des trois questions. Au total, 114 " blocs réponses " ont été analysés ( 3 blocs réponses $\times 38$ enseignants). Les définitions que donnent les enseignants ont été comparées aux définitions institutionnelles de ces notions, relevées dans des textes officiels. Les termes clés présents dans ces textes ont été listés afin d'établir une " définition patron ". L'écart entre les réponses des enseignants et les "définitions patrons » a été mesuré en termes de nombre d'informations recouvertes. Lorsque les réponses des enseignants contenaient plus de $40 \%$ des informations contenues dans les définitions patrons, elles étaient classées "correctes ». Lorsqu'elles contenaient moins de $40 \%$ des informations, elles étaient classées "incorrectes". Certaines réponses, dont aucun élément n'allait dans le sens des textes officiels, ont été classées "erronées ". Un point important est à souligner ici. La confrontation entre les définitions produites par les enseignants et les textes officiels ne permet pas, bien entendu, d'appréhender véritablement les représentations (Abric, 1994). Cela permet toutefois de repérer la connaissance que les enseignants possèdent des textes institutionnels prescriptifs et d'approcher un " certain » point de vue sur les notions travaillées.

Enfin, pour cerner la manière dont les élèves intégrés vivent cette nouvelle modalité de scolarité, les données recueillies auprès d'eux ont fait l'objet d'une analyse logico-sémantique (Bardin, 1989). L'unité retenue est, comme pour la catégorisation précédente, l'ensemble des énoncés (bloc réponse) produits à l'issue de chacune des questions (cf. annexe méthodologique $n^{\circ} 2$ ). Ces réponses peuvent se décliner chez de jeunes élèves d'un seul mot à un ensemble de propositions. Elles ont été classées en trois catégories: "expérience échouée ", "expérience réussie ", " expérience très réussie » (4). Les énoncés regroupés dans la catégorie "expérience échouée " témoignent d'un échec subjectif de l'intégration: "Comment tu te sens dans ta classe ? Pas très bien du tout. Est-ce que tu participes plus à l'oral que l'an dernier ? Non, je n'aime pas parler en classe. D'après toi, est-ce que tes résultats se sont améliorés ? Non, c'est vraiment pareil. ". Ceux classés dans la catégorie " expérience réussie " témoignent à l'inverse d'un vécu positif de l'intégration: "Comment tu te sens dans ta classe ? Bien, oui bien, ça va, tranquille. Estce que tu participes plus à l'oral que l'an dernier? Oui, plus, c'est plus facile. D'après toi, est-ce que tes résultats se sont améliorés ? Oui, c'est mieux, c'est plus facile de travailler. "Enfin, ont été regroupés 
Tableau 1. - Typologie des gestes d'aide mis en œuvre par les enseignants du $\mathbf{1}^{\mathrm{er}}$ et $\mathbf{2}^{\text {nd }}$ degré pour les élèves dyslexiques sévères scolarisés en classe ordinaire (Gombert \& Roussey, 2007)

\begin{abstract}
Catégories et définitions
1. Cadre de travail (énoncés mettant l'accent sur une modification des conditions matérielles de travail dans la classe) :

- Placement de l'élève ;

- Aménagement des conditions matérielles (utilisation d'outils spécifiques et adéquats pour contourner le handicap, aménagements visibles).
\end{abstract}

2. Adaptation de la prescription (énoncés dont l'idée témoigne d'une réflexion de l'enseignant sur la prescription, c'est-à-dire les consignes de mise au travail) :

- Consignes prises en charge par l'enseignant;

- Consignes prises en charge par les élèves.

3. Adaptation des moyens (différenciation pédagogique) : aides propres à compenser le handicap spécifique en lecture et écriture ou résultant de difficultés associées (lenteur d'exécution, non-autonomie cognitive). II s'agit ici, à objectif commun, de prendre en compte la particularité de l'élève concerné en aménageant les conditions pour y parvenir.

- Adaptations en rapport à l'activité de lecture : contournement de l'activité et/ou adaptation des supports;

- Adaptation en rapport avec l'activité d'écriture : contournement de l'activité d'écriture et/ou adaptation des supports ;

- Adaptation en rapport au temps de travail ;

- Modification du style pédagogique de l'enseignant.
Élève devant ou plus près du maître...

Utilisation d'un fil numérique en mathématiques, d'un aide-mémoire orthographique, mise à disposition de boîtes de rangement.
Relecture, explicitation, simplification.

Relecture, reformulation des consignes par l'élève dyslexique, relecture des consignes par un autre élève non dyslexique.
Dispense activité lecture à haute voix, lecture prise en charge par un pair ou par l'enseignant, textes photocopiés, typographie adaptée...

Mise à disposition d'un secrétaire, réduction activité prise de notes, photocopies des cours, augmentation travail sur le manuel scolaire, utilisation de fiches à compléter, textes à trous, étiquettes...

Augmentation du temps de réalisation d'exercices en classe ou à la maison.

Cours plus oralisé, accent mis sur l'expérimentation, travaux pratiques, démonstration, l'exemplification.

4. Adaptation des parcours: individualisation regroupe les énoncés qui mettent l'accent sur la mise en place d'une individualisation de l'enseignement, c'est-àdire, autoriser l'élève à ne pas faire la même chose que ses camarades.

- Réduction de la somme des savoirs ou des notions à faire acquérir ;

- Choix de matières à enseigner.

5. Aide des pairs de la classe vers l'élève handicapé (aide institutionnalisée).

- Travail en groupe ;

- Mise en place de tutorat.

6. Guidance et contrôle individualisé de l'enseignant lors de la réalisation d'une tâche :

regroupe les énoncés qui témoignent d'une aide individualisée conduite par l'enseignant pendant son cours ou d'un " regard/contrôle » plus important du travail de l'élève en cours de réalisation. II s'agit ici de ce que Bruner (1996) nomme le maintien de l'orientation.

Programme à la carte : travail sur fichier lecture CE2 pour élève de CM2.

Dispense d'une ou 2 matières.

Illustrations?

Répétition des consignes et vigilance accrue, circuler plus dans la classe, tapoter sur le bureau pour « capter » l'attention.

Explications supplémentaires individuelles. 
7. Apports méthodologique et métacognitif :

regroupe les énoncés qui témoignent d'un travail effectué dans le domaine des compétences transversales ou métalinguistiques, qui a pour fonction de «signaler les caractéristiques déterminantes " pour la réalisation d'une tâche (Bruner, 1996).

\section{Adaptation à l'évaluation :}

regroupe les énoncés qui témoignent d'une modification des gestes propres à évaluer l'élève que ce soit au moment des contrôles ou lors de la notation elle-même.

- Dans la réalisation du contrôle ;

- Dans la notation.

9. Revalorisation de l'élève :

regroupe les énoncés qui témoignent d'un travail effectué propre à revaloriser, motiver l'élève en difficulté.
Travail sur la tenue de cahier de texte, sur la gestion des devoirs à faire...

Verbalisation des étapes du travail à réaliser et réaliser.

Répétition, simplification des questions/consignes, modification des supports (texte à trous), baisse du nombre d'exercices à réaliser, temps supplémentaire de réalisation accordé, modification des conditions de passation (oral et prise de notes par l'enseignant), contrôle attentif durant l'interrogation, mise à disposition d'un secrétaire.

Notation sur des critères précis, focalisation sur le raisonnement, évaluation formatrice: revenir sur les fautes et les points positifs.

Encouragements, renforcements positifs, travail sur le statut de l'erreur...

10. Absence d'adaptation.

11. Réponses inclassables:

regroupe les réponses des enseignants déclarant ne pas avoir adapté leurs méthodes d'enseignement ou ne pouvant pas être classées car trop imprécises.

dans la catégorie "expérience très réussie» les énoncés qui, grâce à des modalisateurs d'intensité comme "beaucoup, très, largement... » (Moeschler, 1985), manifestent un réel succès de l'intégration pour l'élève : "Comment tu te sens dans ta classe? Vraiment très bien, c'est super cette année. Est-ce que tu participes plus à l'oral que l'an dernier ? Beaucoup plus, c'est vraiment plus facile de lever le doigt, on rigole pas quand je parle. D'après toi, est-ce que tes résultats se sont améliorés? Eh bien, oui, vachement, c'est bien mieux. ". À l'issue de cette catégorisation, chacun des élèves s'est vu attribuer deux indices de réussite du processus intégratif : l'un sur la dimension scolaire (questions du type : "D'après toi, est ce que tes résultats se sont améliorés ? Dans quelles matières ? "), l'autre sur la dimension sociale de leur expérience d'intégration (questions du type : "Comment tu te sens dans ta classe?").

Pour finir, le caractère objectif de la typologie des gestes d'aide (tableau 1) et des deux catégorisations (points de vue des enseignants et vécu de l'expérience scolaire des élèves) a été testé à l'aide de la méthode des juges (Plake, Hambleton, Jaeger, 1997) (voir annexe méthodologique $n^{\circ} 3$ ).

\section{RÉSULTATS ET INTERPRÉTATIONS}

\section{Des profils pédagogiques d'adaptation contrastés?}

Une analyse factorielle en composantes principales mettant en corrélation les différents gestes d'aide déclarés par les enseignants a permis de dégager quatre "profils" de gestes pédagogiques d'adaptation (tableau 2 (5)).

Le premier profil regroupe les gestes principalement centrés sur le niveau cognitif de l'élève. Les enseignants déclarent individualiser les apprentissages et 


\section{Tableau 2. - Corrélation entre les différents types de gestes d'aide mis en œuvre par les enseignants du $1^{\mathrm{er}}$ et $2^{\text {nd }}$ degré pour les élèves dyslexiques sévères scolarisés en classe ordinaire (analyse en composantes principales) (6)}

\begin{tabular}{|c|c|c|c|c|}
\hline Typologie des gestes d'aide & $\begin{array}{c}\text { Profil } 1 \\
\text { - inclusif - } \\
\text { gestes centrés } \\
\text { sur le niveau } \\
\text { cognitif de l'élève }\end{array}$ & $\begin{array}{c}\text { Profil } 2 \\
\text { - intégratif - } \\
\text { gestes centrés } \\
\text { sur les } \\
\text { apprentissages }\end{array}$ & $\begin{array}{c}\text { Profil } 3 \\
\text { - motivationnel - } \\
\text { gestes centrés } \\
\text { sur les aspects } \\
\text { motivationnels }\end{array}$ & $\begin{array}{c}\text { Profil } 4 \\
\text { - attentionnel - } \\
\text { gestes centrés } \\
\text { sur les aspects } \\
\text { attentionnels }\end{array}$ \\
\hline Cadre de travail & & & & ,61 \\
\hline Adaptation consignes & & & ,78 & - \\
\hline Différenciation pédagogique & & ,90 & - & - \\
\hline Individualisation & ,75 & - & - & - \\
\hline Aide des pairs & ,80 & - & - & - \\
\hline Guidance & & & & ,81 \\
\hline Apports méthodologiques &,- 43 & ,41 &,- 53 &, 51 \\
\hline Adaptation évaluation &,- 81 &, 57 & ,43 & - \\
\hline Revalorisation & & & ,67 & - \\
\hline Absence d'adaptation & &,- 64 & - &,- 52 \\
\hline$\%$ variance expliquée & 21 & 17 & 16 & 17 \\
\hline
\end{tabular}

mettre en place régulièrement l'aide entre pairs. Ils partiraient donc du potentiel d'apprentissage des élèves lié à leur handicap, plus qu'ils ne chercheraient à les conduire vers une norme scolaire. En clair, ils s'adapteraient à eux, plus qu'ils ne leur demanderaient de s'adapter aux cours dispensés, en élaborant, par exemple, un programme « à la carte » à partir du programme scolaire. Ils tiendraient ainsi compte des besoins éducatifs particuliers de ces élèves, au quotidien, dans la préparation de leurs séances comme dans les démarches d'apprentissage mises en œuvre dans la classe. Par ailleurs ces enseignants disent adapter très peu les évaluations. Ce type pédagogique d'adaptation tendrait vers le modèle inclusif de scolarisation des élèves en situation de handicap.

Le deuxième profil fait davantage référence au modèle intégratif de scolarisation des élèves en situation de handicap, les gestes étant principalement centrés ici sur les apprentissages. La différenciation pédagogique (adaptation des supports, des activités ou des temps de lecture et d'écriture), l'adaptation des évaluations (hiérarchisation différente des critères de notation, réduction du nombre d'exercices à faire, temps supplémentaire alloué), l'aide méthodologique (aider l'élève à repérer les étapes d'un exercice ou à gérer les différentes tâches de sa scolarité), amènent les élèves par divers moyens vers les apprentissages du groupe classe. Cela les ramène donc vers la norme scolaire de la classe ordinaire. Le mouvement d'incorporation de l'élève dyslexique sévère serait inverse à celui des pratiques décrites dans le profil 1 .

Les gestes d'adaptation pédagogique du troisième groupe portent sur l'adaptation des consignes, des évaluations, ainsi que sur les pratiques de valorisation. Le professeur dit encourager l'élève, mettre en exergue ses réussites plutôt que ses échecs, dédramatiser ses erreurs... Les aspects motivationnels de l'apprentissage semblent davantage mis en exergue ici.

Les gestes regroupés dans le quatrième groupe font référence à la modification du cadre de travail de la classe (placement de l'élève dans la salle, outils mis à disposition), aux aides méthodologiques, et aux pratiques de guidance dans la réalisation de la tâche (répéter les consignes à l'élève, donner des explications supplémentaires, regarder où il en est). L'ensemble de ces gestes professionnels viserait plutôt à rendre l'élève attentif tout au long de la tâche scolaire. 
Se dégagent ainsi quatre profils pédagogiques d'adaptation : le profil « inclusif », le profil « intégratif », le profil « motivationnel », le profil « attentionnel ».

Une chose est de dégager des profils pédagogiques d'adaptation à partir de l'analyse des gestes d'aide. Une autre chose est de tenter de les mettre en lien avec les fonctions de l'étayage telles que Bruner (1983) les propose (cf. § 2.). La tâche n'est pas aisée dans la mesure où des gestes différents peuvent exercer la même fonction et inversement, un même geste peut avoir plusieurs fonctions (par exemple, adapter l'évaluation peut avoir pour but de réduire les degrés de liberté et/ou de contrôler la frustration). Pourtant, on voit bien se dessiner au travers de ces profils des fonctions convergentes.

Le profil "attentionnel " rassemble des gestes qui auraient largement pour fonction de capter et soutenir l'attention des élèves, donc de maintenir l'orientation. En mettant l'accent sur l'adaptation des consignes et de l'évaluation tout en valorisant l'élève, les gestes du profil "motivationnel » exerceraient plutôt une fonction d'enrôlement dans la tâche et de contrôle de la frustration. Enfin les profils " intégratif » et " inclusif », quant à eux, regroupent tous deux des gestes visant, en priorité, à réduire les degrés de liberté et à contrôler la frustration. En effet, partir du potentiel des élèves en individualisant les apprentissages (profil inclusif), ou bien différencier les moyens d'accès au savoir (profil intégratif), concourent bien à la même fonction : simplifier la tâche pour la mettre à portée de l'élève en difficulté à l'écrit. Ce qui varie ici, ce n'est donc pas la fonction des aides mais bien le rapport à la norme de la classe. Dans un cas il s'agit d'amener l'élève vers les apprentissages qu'il n'a pas pu effectuer jusqu'à présent et ce, sans être forcément synchrone avec le groupe classe. Dans l'autre cas, il s'agit plutôt de le ramener vers la norme scolaire de la classe.

\section{Des enseignants du primaire et du secondaire à profil différent ?}

Les enseignants du primaire et du secondaire se répartissent-ils de la même manière entre les quatre profils, ou bien remarque-t-on des différences selon le niveau d'enseignement? Deux analyses ont été conduites pour répondre à ces questions. La première porte sur les gestes d'aide, considérés indépendamment (figure 1). La deuxième porte sur les profils pédagogiques d'adaptation, associant différents gestes professionnels (figure 2).
Figure 1. - Répartition des gestes d'aide mis en œuvre par les enseignants pour les élèves dyslexiques sévères scolarisés en classe ordinaire, selon le niveau d'enseignement

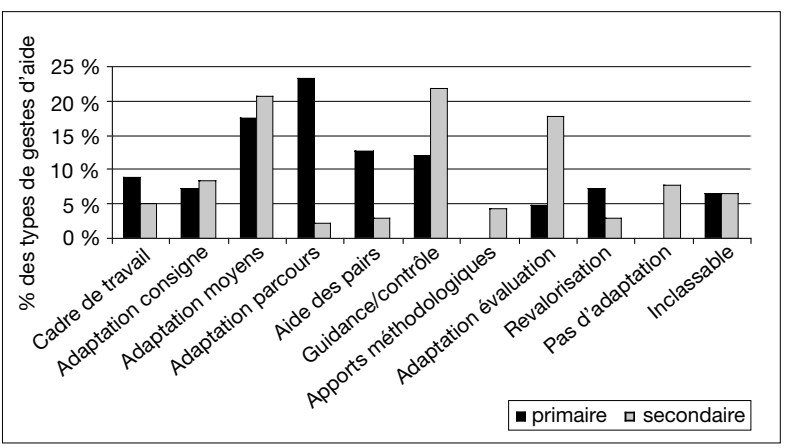

Note: $N=266$ énoncés référents aux gestes dont 125 pour les professeurs d'école et 141 pour les enseignants de collège. Le $\mathrm{X}^{2}$ est significatif pour l'adaptation du parcours, l'aide des pairs, la guidance/contrôle, l'adaptation des évaluations.

Les enseignants du primaire déclarent mettre en œuvre plus de gestes d'aide que ceux du secondaire (7). Néanmoins, de façon globale, les gestes d'aide les plus cités sont similaires dans le primaire et dans le secondaire. Les gestes professionnels le plus souvent évoqués sont ceux qui portent sur l'adaptation des moyens. La différenciation pédagogique des supports et des activités est mise en place aussi bien à l'école qu'au collège (mise à disposition de l'élève d'un secrétaire, réduction de l'activité de prise de notes...). Les gestes pédagogiques les moins évoqués concernent l'adaptation du cadre de travail, de la consigne, les apports méthodologiques et la valorisation de l'élève. Au-delà de ce "fonds commun " de pratiques, partagé, les enseignants du primaire et du secondaire se distinguent de façon significative sur certains types d'aide mise en place (figure 1). Les professeurs d'école disent individualiser davantage les contenus appris, donc les parcours au sein des programmes. Les élèves dyslexiques seraient ainsi autorisés à ne pas faire la même chose que leurs camarades. Ils utilisent également les potentialités du groupe classe comme une aide pour l'élève intégré (tutorat, travail en groupes...). Les enseignants du secondaire déclarent plus que leurs homologues adapter les évaluations et exercer un contrôle individualisé de la tâche en cours. Les étayages proposés relèvent d'un registre différent. Ils concernent les modalités d'apprentissage plus que les savoirs enseignés.

L'analyse des profils pédagogiques d'adaptation confirme ces résultats (figure 2). Le profil intégratif qui 
regroupe les gestes de différenciation pédagogique est associé aux enseignants du primaire tout comme à ceux du secondaire (différence non significative). En revanche, le profil inclusif est quasi exclusivement relié aux professeurs des écoles (8).

Figure 2. - Répartition des profils pédagogiques d'adaptation selon le niveau d'enseignement des enseignants (9)

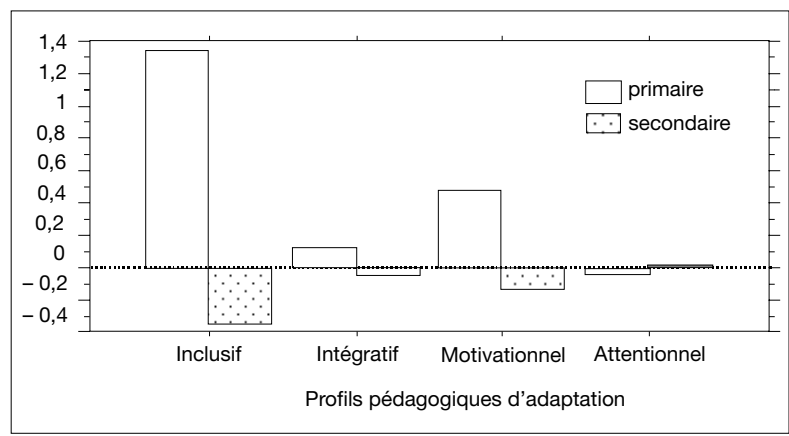

Note: Les colonnes au-dessus de la droite montrent une corrélation positive alors que celles au-dessous témoignent d'une corrélation négative. Par exemple, le " profil inclusif " est surtout associé aux enseignants du primaire et très peu aux enseignants du secondaire.

\section{Profils pédagogiques d'adaptation et points de vue des enseignants}

Globalement, tous les enseignants ont le même point de vue. Ils donnent une définition de l'intégration proche des textes officiels. Les définitions des professeurs des collèges sont également, en majorité, conformes aux textes du handicap et de la dyslexie (tableau 3). Ce n'est pas le cas en revanche de celles des professeurs des écoles, plutôt incorrectes de ce point de vue.

Seules deux corrélations sont significatives. Les enseignants qui possèdent une définition "Correcte " de l'intégration déclarent activer les gestes centrés sur les apprentissages. Ils disent mettre en place une pédagogie différenciée ciblée sur l'adaptation des supports. Ce constat corrobore la typologie des profils pédagogiques, qui établit un lien entre ce profil de pratiques et le modèle intégratif de scolarisation des élèves en situation de handicap. Par ailleurs, les enseignants qui donnent une définition "correcte " de la dyslexie sont ceux qui individualisent le moins les parcours. Ce résultat n'est qu'en apparence paradoxal et peut être expliqué de deux manières. Premièrement, il peut être lié à la faiblesse de l'échan-

Tableau 3. - Degré de conformité entre les définitions des enseignants et les définitions officielles de l'intégration, du handicap, de la dyslexie, selon le niveau d'enseignement

\begin{tabular}{|l|c|c|c|c|c|c|}
\hline & \multicolumn{2}{|c|}{ Définition. Intégration } & \multicolumn{2}{c|}{ Définition. Handicap } & \multicolumn{2}{c|}{ Définition. Dyslexie } \\
\hline & Correcte & Incorrecte & Correcte & Incorrecte & Correcte & Incorrecte \\
\hline Primaire & $\mathbf{8}(100 \%)$ & 0 & 2 & $\mathbf{6}(75 \%)$ & 3 & $\mathbf{5}(62,5 \%)$ \\
\hline Secondaire & $\mathbf{2 8}(93,3 \%)$ & 2 & $\mathbf{1 9}(63,3 \%)$ & 11 & $\mathbf{1 8}(60 \%)$ & 13 \\
\hline
\end{tabular}

Tableau 4. - Corrélation entre les définitions correctes des enseignants (intégration, handicap, dyslexie) et les profils pédagogiques d'adaptation

\begin{tabular}{|l|c|c|c|}
\hline Note : $r$ de Bravais-Pearson & $\begin{array}{c}\text { Définition } \\
\text { Correcte } \\
\text { de l'intégration }\end{array}$ & $\begin{array}{c}\text { Définition } \\
\text { Correcte } \\
\text { du handicap }\end{array}$ & $\begin{array}{c}\text { Définition } \\
\text { Correcte } \\
\text { de la dyslexie }\end{array}$ \\
\hline $\begin{array}{l}\text { Profil 1 : inclusif } \\
\text { Gestes centrés sur le niveau cognitif de l'élève }\end{array}$ & - & - &,- 34 \\
\hline $\begin{array}{l}\text { Profil 2 } \text { intégratif } \\
\text { Gestes centrés sur les apprentissages }\end{array}$ &, 31 & - & - \\
\hline $\begin{array}{l}\text { Profil 3 : motivationnel } \\
\text { Gestes centrés sur les aspects motivationnels }\end{array}$ & - & - & \\
\hline $\begin{array}{l}\text { Profil 4 : attentionnel } \\
\text { Gestes centrés sur les aspects attentionnels }\end{array}$ & - & - & \\
\hline
\end{tabular}


tillon puisque ce sont les enseignants du secondaire qui donnent majoritairement une définition correcte de la dyslexie (18 vs 5). Deuxièmement, le trouble spécifique sévère du langage écrit se "manifeste par d'importantes difficultés dans l'apprentissage de la lecture (dyslexie) ou de l'écriture (dysorthographie) chez des enfants ayant, par ailleurs, une efficience intellectuelle normale » (ONL, 1998). Les enseignants du secondaire sembleraient avoir tout à fait saisi cette nuance et avoir pris en quelque sorte " au pied de la lettre " cette caractéristique. Tout se passe comme s'ils estimaient que ces élèves, grâce à leur efficience intellectuelle préservée, peuvent accéder au même programme que leurs camarades, et ce malgré leur retard scolaire.

\section{Profils pédagogiques d'adaptation et vécu de l'intégration chez l'élève}

Très peu d'élèves ont un sentiment d'échec concernant leur intégration en classe ordinaire (tableau 5). 3 élèves sur 17 seulement l'expriment (2 par rapport à la dimension sociale, 1 par rapport à la dimension scolaire). Les élèves témoignent donc en majorité d'une expérience réussie, très souvent sur les deux dimensions à la fois, scolaire et sociale.

Observe-t-on un lien entre le profil pédagogique d'adaptation des enseignants et le vécu de l'intégration chez les élèves ? Les analyses ne montrent pas de corrélation significative entre ces deux aspects. L'effectif très faible d'élèves ayant un sentiment d'échec (3 au total) explique sans aucun doute ce résultat. Cette modalité n'étant quasiment pas représentée, cela peut réduire d'autant les effets possibles des gestes enseignants sur cette variable. Cependant, deux constats sont à relever (comme étude de cas), éclairés par l'analyse de contenu des entretiens individuels des élèves concernés et par d'autres éléments d'évaluation.
En ce qui concerne la dimension scolaire (figure $3 a$ ), les enseignants de l'élève qui a un sentiment d'échec face à ses apprentissages font partie de ceux qui mettent le moins en œuvre les gestes propices à maintenir l'attention dans la tâche scolaire. Ils n'adaptent pas ou peu le cadre de la classe, ne contrôlent pas ou peu la réalisation de l'exercice, ne mettent pas ou peu l'accent sur les techniques de travail (apports méthodologiques). Or, on peut penser que ces gestes génériques simples (mettre l'élève devant, tapoter sur la table lorsqu'il s'échappe de l'activité, circuler dans la classe) sont essentiels pour cet élève. En effet, les résultats que cet élève avait obtenus lors de la passation de la «nepsy » (bilan neuropsychologique de l'enfant, Korkman, Kirk, Sally Kemp, 2003) en début d'année scolaire, montrent des compétences attentionnelles largement plus altérées chez lui que chez la plupart des autres élèves dyslexiques intégrés dans ce dispositif de scolarisation.

Figure 3a. - Corrélation entre les profils pédagogiques d'adaptation des enseignants et le vécu de la dimension scolaire de l'intégration chez les élèves (10)

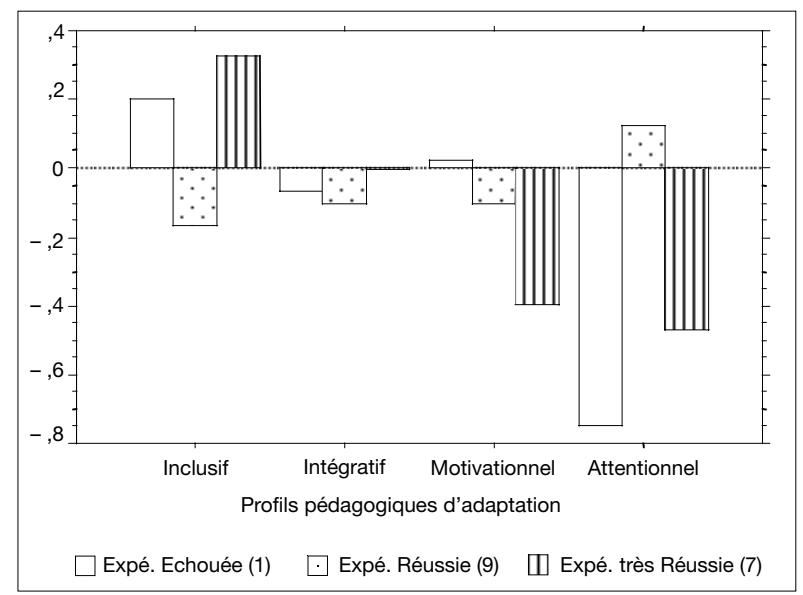

Tableau 5. - Vécu des dimensions scolaire et sociale de l'intégration chez les élèves (en effectifs)

\begin{tabular}{|l|l|c|c|c|c|}
\hline \multicolumn{2}{|c|}{} & Échouée & Réussie & Très réussie & Total \\
\hline \multirow{4}{*}{$\begin{array}{l}\text { Dimension } \\
\text { scolaire }\end{array}$} & Échouée & 0 & 1 & 0 & 1 \\
\cline { 2 - 6 } & Réussie & 2 & 6 & 1 & 9 \\
\cline { 2 - 6 } & Très réussie & 0 & 0 & 7 & 7 \\
\cline { 2 - 6 } & Total & 2 & 7 & 8 & 17 \\
\hline
\end{tabular}




\section{Figure 3b. - Corrélation entre les profils pédagogiques d'adaptation des enseignants et le vécu de la dimension sociale de l'intégration chez les élèves}

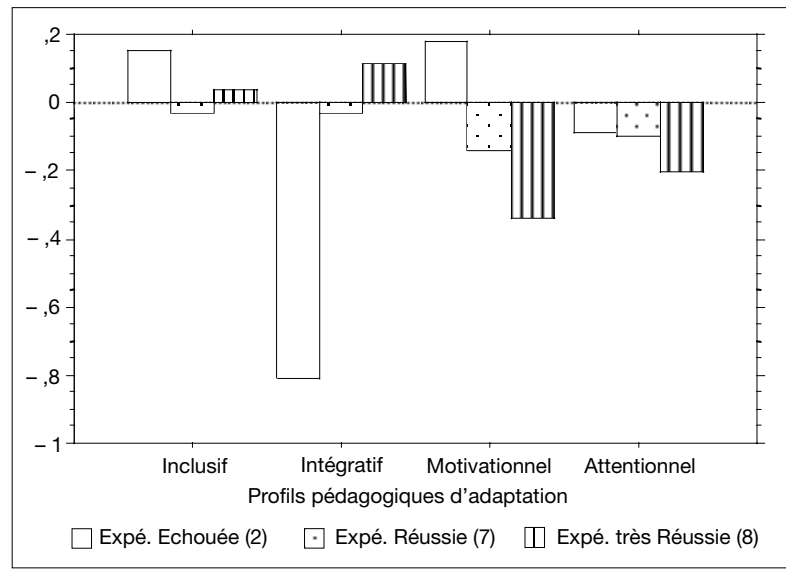

En ce qui concerne la dimension sociale (figure 3b), les enseignants des deux élèves qui se sentent mal dans la classe, ne participent pas à l'oral, n'observent pas de différence positive par rapport à leur scolarité antérieure, mettent moins en œuvre les gestes de différenciation pédagogique. Une enquête en cours traitant également de cette question, sur une scolarisation à plein temps en classe ordinaire et non plus à mi-temps, permettra de corroborer ou non ce constat. La non-adaptation des supports d'apprentissage laisserait-elle penser à ces élèves que l'enseignant ne prend pas en compte leurs différences, et réduiraitelle leur sentiment d'appartenance au groupe classe ainsi qu'au dispositif ?

\section{Discussion}

La recherche présentée se proposait d'éclairer trois aspects de la scolarisation en classe ordinaire d'élèves dyslexiques sévères. Dans un premier temps, il s'agissait de cerner les pratiques d'aide des professeurs du primaire et du secondaire; dans un deuxième temps, de voir si la manière de concevoir le handicap, l'intégration, ou la dyslexie chez les enseignants a un impact sur leurs pratiques ; enfin, d'examiner si les profils pédagogiques d'adaptation sont en lien avec la manière dont les élèves dyslexiques vivent leur intégration.

En ce qui concerne les gestes d'aide des enseignants, l'enquête a permis de mettre en évidence la modification de certains gestes pédagogiques "simples » (par exemple: placer un élève devant, l'autoriser à moins prendre de notes, lui distribuer des photocopies du cours, lui relire les consignes écrites, simplifier les consignes et la présentation des exercices) dont le but est de permettre aux élèves dyslexiques sévères de suivre les apprentissages de la classe, alors même qu'ils ont de grandes difficultés avec l'écrit et que la plupart de ces apprentissages passe par l'écrit. De fait, d'après les dires des enseignants, il s'agit d'adapter leurs manières de faire en cours afin de compenser ces difficultés d'accès à l'écrit, spécifiques de leur trouble. Les gestes d'aide cités représentent bien des adaptations pédagogiques qui constituent, dans ce cadre, un moyen de compenser les conséquences du handicap de ces élèves.

À cet égard le premier constat qui peut être fait à partir des résultats est que tous les enseignants impliqués dans cette expérimentation, quel que soit leur niveau d'enseignement, disent faire de la différenciation pédagogique dans le but d'aider les élèves intégrés à surmonter leurs difficultés en s'appuyant notamment sur l'adaptation des supports d'apprentissage. D'autres types de gestes semblent plus spécifiques et varient en fonction du niveau d'intervention des professeurs. C'est le cas, par exemple, de l'adaptation de l'évaluation (évaluation critériée, adaptations des supports) qui apparaît comme l'apanage des enseignants du second degré, tout comme les gestes de guidance mise en œuvre dans le but de maintenir l'élève dans la tâche qu'il est en train d'accomplir. Ce souci a amené les enseignants du second degré, par exemple, à circuler plus dans la classe pour contrôler le travail en cours de réalisation, ou à demander à l'élève de lui signifier plus systématiquement sa progression dans le travail qu'il est en train d'accomplir. L'individualisation des apprentissages est plutôt l'affaire des enseignants du primaire qui, en réduisant la quantité des savoirs et notions à acquérir, semblent privilégier le niveau réel des élèves intégrés plutôt que leur niveau scolaire théorique.

La focalisation des professeurs de collège sur la dimension évaluative et leur frilosité à individualiser les apprentissages proposés aux élèves intégrés pourraient concrétiser une préoccupation spontanée majeure, qui consiste à amener l'élève le plus près possible de la norme institutionnelle afin qu'il puisse, en bout de course, valider un diplôme. Comme le notent Gombert et Roussey (2007) «les enseignants du second degré, plus que leurs homologues du premier degré, se trouveraient dans une situation paradoxale, tiraillés entre la mission qui leur est fixée, consistant à prendre en charge l'élève à besoins particuliers intégré 
dans sa différence jusqu'à prendre en compte ce qu'il ne peut pas faire à cause de son trouble, et leur désir de l'amener au terme du curriculum scolaire normal défini par les programmes et les textes institutionnels ». En effet, même si ces textes ont largement évolué ces cinq dernières années et rendent maintenant possible un aménagement des conditions d'examen pour les élèves handicapés (tiers temps supplémentaire, mise à disposition d'un secrétaire ; BOEN $n^{\circ} 13 \mathrm{du}$ 31 mars 2006), il n'est toujours pas possible, contrairement à ce qui se fait par exemple en Italie, d'établir une modulation curriculaire permettant de passer un examen sur la base d'un programme individualisé (De Anna, 2003). Dans ce cadre, il n'est donc pas étonnant que l'individualisation, dans le second degré, soit un geste peu évoqué. Cette interprétation en référence à la norme institutionnelle prescrite en amène une autre autour de la notion de justice scolaire. Donner à un élève autre chose à faire que ses camarades nécessite de concevoir que l'inégalité de parcours peut être équitable et donc porteuse d'égalité d'apprentissage. Cette idée est davantage " digérée " pour un enseignant du premier degré probablement grâce à sa formation plus importante dans le domaine des compétences interdisciplinaires et transversales, à son contexte de travail favorisant la prise en charge de l'hétérogénéité (cycle, décloisonnement...), et à sa plus longue expérience de la prise en compte du handicap (création des CLISS dès 1983). Les données d'une enquête en cours permettront de vérifier si effectivement il existe bel et bien une différence de représentation de l'équité scolaire entre enseignant du primaire et secondaire.

Quatre profils pédagogiques d'adaptation représentant quatre combinaisons différentes de ces gestes d'aide émergeant des analyses. Ainsi, lorsque les enseignants évoquent des gestes d'individualisation, ils évoquent aussi l'aide entre pairs (profil inclusif). Le profil « intégratif » regroupe principalement les gestes d'adaptation des supports ; le profil « motivationnel " rassemble les gestes d'encouragement avec ceux qui modifient les formes scolaires pouvant mettre l'élève en échec (adaptation de consignes, évaluations adaptées, statut de l'erreur) ; le profil « attentionnel » combine les gestes qui portent plutôt sur l'aménagement des conditions de travail (modification du cadre matériel) avec ceux qui ont pour vocation de maintenir l'élève dans l'activité. La répartition des enseignants parmi ces profils varie selon le niveau d'enseignement pour le profil inclusif seulement, que l'on retrouve presque exclusivement chez les professeurs des écoles primaires. Ainsi, les professeurs des écoles comme ceux de collège réduisent la difficulté sco- laire en diminuant les degrés de liberté, c'est-à-dire en décomposant puis en recomposant autrement les activités d'apprentissage afin de ne pas surcharger l'élève, tant au niveau des capacités qu'il doit mobiliser pour effectuer la tâche, qu'au niveau des informations présentes dans cette tâche. Ce qui les différencie en revanche, c'est la manière dont ils disent mettre en œuvre cette différenciation pédagogique (cf. paragraphe précédent).

La mise en cuvre de gestes d'aide envers un élève en situation de handicap, la dyslexie sévère en l'occurrence, constitue un véritable défi pour les professeurs. II s'agit d'une part de savoir "quoi faire", quels étayages mettre en place à un moment donné. D'autre part il s'agit de conduire une réflexion sur le "comment faire ", sur la manière de rendre l'élève autonome par rapport à ces aides. La culture de l'étayage n'est pas une évidence dans le paysage éducatif français et ces adaptations pédagogiques sont une évolution des gestes professionnels habituellement mis en œuvre par les enseignants, leur permettant de faire coïncider prescription (scolariser les élèves dyslexiques sévères en difficulté d'apprentissage) et réalisation de leur activité quotidienne (apprendre le programme au groupe classe et le faire progresser). L'analyse de contenu de leurs réponses à une autre question posée dans les entretiens (non présentée dans l'article) : "Comment définiriez-vous votre rôle d'enseignant du point de vue de l'intégration ? ", montre que les adaptations pédagogiques, à travers l'ébauche et l'invention de nouvelles manières de faire, transforment cette activité. Plusieurs enseignants disent que ces évolutions modifient leur façon de voir. Certains évoquent que les adaptations destinées au départ aux élèves dyslexiques servent à toute la classe, et leur permettent de repenser les cours pour les autres élèves en difficulté (mais non porteurs de handicap). Celles-ci deviennent des ressources. Les profils qui en découlent peuvent être mis en relation avec la notion de genre professionnel développée par Clot \& Faïta (2000). Le « genre » définit l'ensemble des façons de faire identifiables qui se constitue dans l'histoire d'une activité professionnelle, et qui devient une ressource, pour le professionnel qui l'a développé ou pour d'autres. Cet ensemble de pratiques a vocation à se transformer (régulations, modifications des gestes), puisqu'il se forme au cœur même de l'activité. II s'agit donc plus de capacités d'adaptation aux circonstances de travail, de dispositions pratiques, que de routines pouvant s'automatiser. La méthodologie de l'enquête présentée ici ne permet pas d'affirmer si ces profils constituent des ébauches de genres professionnels. Néanmoins, ces 
résultats ouvrent une piste de réflexion sur l'impact de la scolarisation en classe ordinaire d'élèves ayant des troubles spécifiques d'apprentissage en langage écrit, sur la transformation de l'activité enseignante.

L'impact des définitions des enseignants concernant l'intégration, le handicap ou la dyslexie, sur les pratiques d'aide, est partiel. Deux corrélations seulement sont établies : (a) avoir une définition "correcte " de l'intégration, eu égard aux textes officiels, entraîne des gestes d'adaptation des supports d'apprentissage ; (b) avoir une définition « correcte » de la dyslexie implique rarement l'individualisation des parcours. Même si ces résultats sont à moduler compte tenu des faibles effectifs, ils soulèvent des pistes intéressantes. Dans les deux cas, la mise en œuvre pédagogique est cohérente par rapport à la manière de concevoir la scolarisation des élèves porteurs d'un trouble du langage écrit. Le modèle intégratif suppose un soutien de l'enseignant, permettant à l'élève qui possède des capacités sociales et scolaires, de rejoindre le groupe classe et les difficultés de lecture et d'écriture de ces élèves s'accompagnent toujours d'une efficience intellectuelle normale.

L'enquête n'établit pas d'impact des profils pédagogiques d'adaptation des enseignants sur la manière dont les élèves dyslexiques vivent leur intégration (Gombert, Feuilladieu, Gilles, Roussey, 2007). Presque tous ont un ressenti positif concernant cette modalité de scolarité au niveau des apprentissages comme de leur intégration sociale (cf. tableau 5: 14 élèves sur 17). Quels que soient les profils de gestes de leurs enseignants, ils se sentent bien en classe, participent plus à l'oral qu'auparavant, trouvent que leurs résultats se sont améliorés, pensent que c'est plus facile d'apprendre. On peut néanmoins penser que les diverses adaptations pédagogiques des professeurs qui les accueillent, destinées à les aider au niveau scolaire, jouent un rôle important dans ce ressenti. D'autres variables ont sans doute aussi joué un rôle. Le dispositif de prise en charge de ces élèves instaure en effet un accompagnement des enseignants par les chercheurs et l'institution, un partenariat entre les différents acteurs, une formalisation du travail au plus près de l'élève dans un projet opérationnel. Même si les données de l'enquête ne permettent pas de montrer l'impact de ces facteurs sur le vécu de cette expérience scolaire par les élèves et la mise en place d'une différenciation pédagogique dans les classes, d'autres enquêtes (Agence européenne, 2003) laissent à penser que ce sont des éléments facilitateurs de l'intégration scolaire des élèves en situation de handicap. La différenciation pédagogique est entendue ici comme "quête d'une activité optimale pour chacun, optimale d'abord sous l'angle de sa praticabilité, de son sens, de sa capacité de mobiliser les élèves concernés, optimale aussi et surtout sous l'angle des apprentissages qu'elle engendre " (Perrenoud, 2001).

Anne Gombert a.gombert@aix-mrs.iufm.fr Centre de recherche en psychologie de la connaissance, du langage et de l'émotion, université de Provence

Sylviane Feuilladieu Apprentissage, didactique, évaluation, formation, université de Provence

Pierre-Yves Gilles

Centre de recherche en psychologie de la connaissance, du langage et de l'émotion, université de Provence

Jean-Yves Roussey

Centre de recherche en psychologie de la connaissance, du langage et de l'émotion, université de Provence

\section{NOTES}

(1) «Le concept de besoin éducatif particulier tient, pour une part à un paradoxe : a priori centré sur l'élève qui aurait des besoins particuliers, il renvoie au dispositif d'enseignement et en particulier à sa capacité à accepter des élèves différents. Le besoin éducatif particulier est donc un concept relatif qui réfère à des questions plus générales comme la gestion de l'hétérogénéité $[. .$. ou encore la laïcité $[. .$.$] en les élargissant à un public particulier :$ minorités culturelles, handicap, maladie... » (Thomazet, 2004).

(2) Circulaire $n^{\circ} 2002-024$, B.O.E.N n 6 du 7 février 2002.

(3) Nous tenons à remercier sincèrement tous les enseignants des écoles primaires et des collèges de Briançon, les principaux, ainsi que le personnel de la MECS pour leur investissement dans ce dispositif.

(4) Les réponses ont été classées en fonction de leur contenu manifeste et de leur valence effective. Autrement dit, aucune analyse de la connotation, ni des sous-entendus, n'a été opérée.
(5) Les 4 profils pédagogiques correspondent aux 4 facteurs mis en évidence par l'analyse, associant certains gestes.

(6) Analyse effectuée avec rotation "varimax " (valeurs propres supérieures à 1). Les énoncés des variables dépendantes ont été codés 0 ou 1 selon la présence ou l'absence des gestes, pour chacun des enseignants.

(7) $(\mathrm{F}(1,36)=27.94 ; \mathrm{p}<.0001: \mathrm{mpe}=5.385 .38 ; \mathrm{mpc}=2.37)$, résultat confirmé par une analyse de la variance (Anova à mesures répétées $)=(F(1,108)=34,7 ; p<.0001)$.

(8) Écarts significatifs uniquement sur le facteur 1.

(9) Note : VD : scores factoriels ; moyenne $=0$; écart-type $=1$.

(10) Note: VD = réponses des enseignants, $0=$ absence, 1 = présence des gestes d'aide; scores factoriels centrés réduits. 


\section{BIBLIOGRAPHIE}

ABRIC J.-C. (1994). Pratiques sociales et représentations. Paris : PUF.

AGENCE EUROPÉENNE POUR LE DÉVELOPPEMENT DE L'ÉDUCATION DES PERSONNES AYANT DES BESOINS PARTICULIERS (2003). Intégration scolaire et pratiques pédagogiques. Rapport disponible sur le site : <www. european-agency.org> (consulté le 12 décembre 2008).

BARDIN L. (1989). L'analyse de contenu. Paris : PUF.

BELMONT B. \& VERILLON A. (2003). Diversité et handicap à l'école: quelles pratiques éducatives pour tous. Paris: INRP/CTNERHI.

BRUNER J.-S. (1983). Le développement de l'enfant, savoir faire, savoir dire. Paris : PUF.

BRUNER J.-S. (1984). «Contextes et formats ». In M. DELEAU (éd.), Langage et communication à l'âge préscolaire. Rennes: Presses universitaire de Rennes.

CLOT Y. \& FAÏTA D. (2000). « Genres et styles en analyse du travail : concepts et méthode ». Travailler, $n^{\circ} 4$, p. 7-42.

DE ANNA L. (2003). "Un enfant gravement handicapé à l'école. L'intégration dans le contexte italien ". In B. BELMONT (dir.) \& A. VÉRILLON (dir.), Diversité et handicap à l'école. Quelles pratiques éducatives pour tous. Paris : INRP/CTNERHI, p. 37-59.

DUBET F. \& MARTUCELLI D. (1996). À l'école. Sociologie de l'expérience scolaire. Paris : Seuil.

FELOUZIS G. (1997). L'efficacité des enseignants. Paris : PUF.

GILLIG J.-M. (2006). Intégrer l'enfant handicapé à l'école. Paris : Dunod.

GOFFMAN E. (1975). Stigmate [1963]. Paris : Minuit.

GOMBERT A. \& ROUSSEY J.-Y. (2007). « L'intégration en classe ordinaire d'élèves souffrant de troubles spécifiques sévères du langage écrit: adaptations pédagogiques des enseignants de collège et de primaire ". Repères, $\mathrm{n}^{\circ} 35, \mathrm{p} .233-251$.

GOMBERT A., FEUILLADIEU S., GILLES P.-Y. \& ROUSSEY J.-Y. (2007). Intégration en classe ordinaire d'élèves porteurs de handicap: pratiques pédagogiques adaptées, représentations des enseignants et expérience scolaire des élèves. Actes du colloque "Les effets des pratiques enseignantes sur les apprentissages des élèves ". Besançon : Pôle Nord-Est des IUFM [CD-Rom].

LAUTIER N. (2001). Psychologie de l'éducation. Regard sur les situations d'enseignement. Paris : Armand Colin.

MOEŚCHLER J. (1985). Argumentation et conversation. Éléments pour une analyse pragmatique du discours. Paris : Hatier/Crédif.

ORGANISATION MONDIALE DE LA SANTÉ (2002). Classification internationale du fonctionnement, du handicap et de la santé. Paris : CTNERHI.

OBSERVATOIRE NATIONAL DE LA LECTURE (1998). Apprendre à lire. Paris : CNDP/Odile Jacob.

PERRENOUD P. (2005). L'école face à la diversité des cultures. La pédagogie différenciée entre exigence d'égalité et droit à la différence. Université de Genève. Disponible sur le site : <www.unige.ch/fapse/SSE/teachers/perrenoud/ php_2001/2001_27.html> (consulté le 12 décembre 2008).

PERRENOUD P. (2001). Individualisation des parcours et différenciation des prises en charge. Université de Genève. Disponible sur le site: <www.unige.ch/fapse/SSE/ teachers/perrenoud/php_2001/2001_27.html>(consulté le 12 décembre 2008).
PESLOUAN D. \& RIVALLAND G. (2003). Guide des aides aux élèves en difficulté. Adaptation et intégration scolaires. Issy-les-Moulineaux : ESF.

PIANTA R.-C., STEINBERG M.-S. \& ROLLINS K.-B. (1995). "The first two years of school: teacher - child relationships and deflections in childrens ". Developpement and Psychopathologie, $\mathrm{n}^{\circ} 7$, p. 295-315.

PLAISANCE E. \& GARDOU C. (coord.) (2001). Dossier : "Situations de handicap et institution scolaire ". Revue française de pédagogie, $\mathrm{n}^{\circ} 134$.

PLAKE B., HAMBLETON R. \& JAEGER R. (1997). «A new standard-setting method for performance assessments: The dominant profile judgment method and some fieldtest results". Educational and Psychological Measurement, vol. $57, \mathrm{n}^{\circ} 3$, p. 400-411.

PLANTIN C. (1993). Lieux communs. Topoï, stéréotypes, clichés. Paris : Kimé.

POIRIER N., GIROUX N., PAQUET A. \& FORGET J. (2005). "L'intégration scolaire des enfants autistes ". Revue de psychoéducation, vol. 34 (2), p. 265-286.

RIVARD M. \& FORGET J. (2006). "Les caractéristiques de l'enfant atteint d'un trouble envahissant du développement en lien avec le degré d'intégration sociale en milieu scolaire ordinaire ". Pratiques psychologiques, $n^{\circ} 12$, p. 271-295.

ROBERTSON K., CHAMBERLAIN B, KASARI C. (2003). « General education teachers' relationship with included students with autism ". Journal of Autism and Developmental disorders, $\mathrm{n}^{\circ} 33$, p. 123-130.

ROUSSEAU N. \& BELANGER S. (2004). La pédagogie de l'inclusion scolaire. Québec: Presses de l'université du Québec.

SCHNEUWLY B. \& BRONCKART J.-P. (1985). Vygotsky aujourd'hui. Paris : Delachaux \& Niestlé.

THOMAZET S. (2006). «De l'intégration à l'inclusion. Une nouvelle étape dans le processus de normalisation de l'école ". Le français aujourd'hui, n¹52, p. 19-27.

THOMAZET S. (2004). "La formation des maîtres dans la perspective d'une prise en compte des besoins éducatifs particuliers ". In Le système éducatif français et les élèves à besoins éducatifs particuliers. Paris : ministère de l'Éducation nationale (À propos de), p. 49-55.

ZAFFRAN J. (1997). L'intégration scolaire des handicapés. Paris : L'Harmattan.

Textes officiels

FRANCE. MINISTÈRE DE L'ÉDUCATION NATIONALE (2006). Élèves handicapés: mise en œuvre et suivi du Projet Personnalisé de Scolarisation. Circulaire $n^{\circ} 2006-126$ du 17 août 2006, BO, n 32,7 septembre 2006.

FRANCE. MINISTÈRE DE L'ÉDUCATION NATIONALE (2002). Mise en œuvre d'un plan d'action pour les enfants atteints d'un trouble spécifique du langage oral ou écrit. Circulaire $n^{\circ} 2002-024$ du 31 janvier 2002, BO $n^{\circ} 6$ du 7 février 2002.

FRANCE (2005). Loi pour l'égalité des droits et des chances, la participation et la citoyenneté des personnes handicapées. Loi 2005-102 du 11 février 2005, JO du 12 février 2005.

FRANCE (1975). Loi d'orientation en faveur des personnes handicapées. Loi d'orientation $\mathrm{n}^{\circ} 75-534$ du 30 juin 1975, JO du 1 juillet 1975. 


\section{ANNEXES MÉTHODOLOGIQUES}

$N^{\circ}$ 1: Grille d'entretien semi-directif des enseignants intégrant des élèves dyslexiques sévères en primaire et dans le secondaire, questions utilisées pour les résultats présentés dans cet article :

- "Vous intégrez dans votre classe un élève dyslexique sévère depuis plus de 3 mois maintenant. Avezvous changé quelque chose dans vos manières de faire, dans vos pratiques pédagogiques?

- Plus spécifiquement, comment prenez-vous en compte ses difficultés ?

- Avez-vous été amené à mettre en place des dispositifs spécifiques pour l'intégrer et l'aider?

- Qu'est-ce qui a été le plus difficile ou le plus facile pour vous?

- Pour vous c'est quoi intégrer?

- Pour vous c'est quoi la dyslexie?

- Pour vous c'est quoi le handicap?»

$N^{\circ} 2$ : Grille d'entretien semi-directif des élèves dyslexiques sévères intégrés à mi-temps en primaire et au collège, questions utilisées pour les résultats présentés dans cet article :

- "Avant que tu intègres ce dispositif de double prise en charge tu étais scolarisé sans prise en charge spécifique de ta difficulté : est-ce que tu vois des différences entre cette année et l'année dernière lorsque tu es en cours?

- Comment tu te sens dans ta classe?
- Est-ce que tu participes plus à l'oral que l'an dernier?

- D’après toi, est ce que tes résultats se sont améliorés? Dans quelles matières?

- Est-ce que c'est plus facile d'apprendre? »

$N^{\circ} 3$ : Validation de la typologie des gestes d'aide des enseignants destinés aux élèves dyslexiques sévères et des catégorisations des énoncés :

"Le caractère objectif de la typologie des gestes d'aide (tableau 1) et des deux catégorisations (représentations et expérience de scolarité) a été testé à l'aide de la méthode des juges (Plake, Hambleton, Jaeger, 1997). La validation du codage et du classement des énoncés a été effectuée grâce à deux juges, qui avaient pour tâche de vérifier que les énoncés étaient classés correctement et ne pouvaient appartenir qu'à une seule catégorie (ou sous catégorie). Si le classement d'un item posait problème trois solutions étaient envisageables: (a) Soit l'énoncé était déplacé pour être reclassé par les deux juges dans une autre et même catégorie ; (b) Soit l'énoncé était reclassé dans l'autre catégorie par un des deux juges ; (c) Soit l'énoncé était reclassé dans deux catégories différentes par les deux juges. Dans ce dernier cas un troisième juge était sollicité. II donnait son avis quant au choix de la catégorie la plus pertinente. L'énoncé était alors reclassé à la majorité. Ce troisième juge n'est intervenu que de façon très ponctuelle (7 fois pour la validation de la typologie et 3 fois pour la catégorisation des représentations). » 